\title{
Dificultades en la relación maternofilial: un caso tratado mediante entrenamiento en asertividad y otras técnicas complementarias
}

\author{
Marta Ortega Otero \\ Ma Elena Aranda Rubio \\ Mateusz Kendzierski \\ Universidad Autónoma de Madrid
}

RESUMEN

El presente trabajo describe la intervención y los resultados obtenidos en un caso de dificultades en la relación maternofilial. Se expone el caso de M., mujer de 48 años con problemas en la relación con su hija adolescente. Se desarrollaron dos sesiones de evaluación, una de explicación del análisis funcional, diecisiete de tratamiento y cinco de seguimiento. Para disminuir la frecuencia de intercambios aversivos entre ambas se llevó a cabo un tratamiento cognitivo conductual basado principalmente en entrenamiento en asertividad, en técnicas de control de activación y en estrategias de afrontamiento. Los resultados obtenidos reflejan una disminución de la frecuencia de intercambios conflictivos y una reducción del malestar en la convivencia.

\section{ABSTRACT}

The present study has the aim of describing the treatment was carried out in a case of mother-to-child difficulties and the analysis of the results of the already said intervention to value its utility. It is exposed the case of M., woman of 48 years old who has problems in her relationship with her daughter. The psychological intervention was made of twenty-five sessions: Two assessment sessions, seventeen cognitivebehavioral sessions and five follow-up sessions. To decrease the frequency of conflicts between them it was carried out a cognitive-behavioral treatment based primarily on assertiveness training, activation control techniques and coping techniques. The results show a decrease in the frequently of aversive exchanges and reduced discomfort in coexistence.
Palabras clave: caso clínico; relación; intercambios conflictivos; entrenamiento en asertividad.
Keywords: Clinical case; relationship; conflicts; assertiveness training. 
El comportamiento de los seres humanos está determinado por los factores del individuo, del ambiente y de la interacción entre ambos. En ocasiones, las personas pueden tener dificultades para interactuar de manera adecuada y adaptada a la necesidad de la situación, por lo que es necesario el aprendizaje de habilidades sociales que les ayude a comportarse de una manera eficaz y adaptativa.

La asertividad es una parte esencial de las habilidades sociales. Uno de los problemas que se encuentra al definir este constructo es delimitar si se trata de un rasgo de personalidad o de una conducta susceptible de ser aprendida; la evidencia científica demuestra que las habilidades sociales se adquieren por medio de experiencias en las relaciones interpersonales. Llanos (2006) afirma que las habilidades sociales pueden aprenderse mediante el proceso de aprendizaje social y tienen un carácter cultural, de modo que están sujetas a las normas sociales del contexto en el que se desarrollan. El modelo conductual enfatiza que la capacidad de respuesta se adquiere y que consiste en un conjunto identificable de características específicas.

Salter fue el primer autor que describió la conducta asertiva en 1949, considerándola un rasgo de personalidad. Sin embargo, Wolpe (1958) y Lazarus (1966) la definieron posteriormente como "la expresión de los derechos y sentimientos personales". Por su parte, Alberti y Emmons (1978) definen la comunicación asertiva como la conducta que permite a una persona actuar en función de sus intereses, defenderse sin ansiedad inapropiada, expresar cómodamente sentimientos honestos o ejer- cer los derechos personales sin negar los de los demás. Asimismo, se entiende que la asertividad es un conjunto de conductas caracterizado por la defensa de los propios intereses, la expresión de las opiniones personales y el respeto de los derechos de los demás (Ortega \& Calero, 2015). Según Roca (2007), los derechos asertivos son aquellos que asumimos que tiene cualquier ser humano, por el hecho de serlo, y son válidos tanto para nosotros como para las demás personas. De esta manera, dado que los derechos son válidos para nosotros mismos y para los demás, también se deben cumplir unas responsabilidades que permitan a la otra persona hacer uso de sus derechos.

En la misma línea, Carrasco (1984) considera que la conducta asertiva tiene cuatro componentes básicos: afirmación de opiniones personales, situación interpersonal, consecución de autorrefuerzos y no utilización de conductas aversivas.

Respecto al primero, se refiere a la ratificación de la forma de pensar y actuar del individuo en cuanto a lo que es o no es adecuado, siendo la persona misma quien marca los criterios en las diferentes situaciones. Por otro lado, aunque es cierto que la persona puede comportarse asertivamente consigo misma, la asertividad suele darse dentro de una situación interpersonal, es decir, dentro de un contexto de naturaleza social en el que el sujeto persigue el objetivo de fomentar una relación positiva con la otra persona, quien a su vez puede reforzar su manera de comportarse. Se habla de autorrefuerzos en el sentido de que cuando el individuo logra emitir una conducta asertiva no siempre va a ser reforzada por su interlocu- 
tor. Asimismo, también su interlocutor puede castigar esa conducta asertiva y hacerle disminuir la frecuencia de emisión de su actuación asertiva en posteriores ocasiones. Por último, el componente de no utilización de conductas aversivas no sólo proporciona mayor posibilidad en la obtención de un refuerzo social, sino que también hace disminuir la probabilidad de futuros problemas en la interacción social.

Retomando la definición del concepto que acontece, la asertividad se entiende como una habilidad que puede ser aprendida y que permite una relación adecuada entre los individuos.

La conducta asertiva se puede explicar desde un punto de vista verbal si tenemos en cuenta el contenido del mensaje y desde un punto de vista no verbal si nos centramos en el estilo del propio mensaje. Igualmente, esta conducta se puede manifestar desde el polo positivo (cuando la persona expresa afecto a su interlocutor) o, por el contrario, desde el polo negativo (en el que la persona es capaz de poner límites y hacer críticas de una manera adecuada). En el polo positivo se encuentra lo que se conoce como aceptación asertiva, siendo ésta la habilidad para expresar cumplidos u opiniones a los demás cuando estos han llevado a cabo una conducta positiva que los justifique. En el polo negativo se sitúa la oposición asertiva, siendo ésta la conducta que lleva a cabo la persona cuando es capaz de oponerse o rechazar la conducta o comentario de su interlocutor utilizando una respuesta apropiada a la situación.

Según Castanyer (2014), las situaciones en las que suele ser necesaria la práctica de la asertividad son: decir no, emitir/recibir críticas, supervisar, expresar sentimientos, manifestar una opinión, discutir y realizar peticiones.

Según Carrasco (1984), existe una serie de factores y circunstancias que podrían ser la base de gran parte de las conductas no asertivas. En primer lugar, los individuos pueden comportarse de forma no asertiva porque no han tenido oportunidad de aprender a comportarse de forma asertiva en una determinada situación (modelo déficit conductual). Por otro lado, la educación y los patrones culturales desempeñan un papel fundamental, ya que las conductas no asertivas pueden haber resultado premiadas y las asertivas castigadas. Estos aprendizajes en los que se refuerza la conducta no asertiva (permitiéndole a la persona obtener ventajas a corto plazo aunque a largo plazo resulte perjudicial), interfieren y dificultan la aparición de conductas asertivas.

Se detallan a continuación los tres estilos de comunicación que se pueden adoptar en toda relación interpersonal. Esta descripción se basa en los trabajos realizados por Bueno y Garrido (2012), así como Caballo (1997), que clasifican los estilos de comunicación en agresivo, pasivo/inhibido y asertivo.

El estilo agresivo es aquel que lleva a cabo la persona que utiliza conductas que agreden a los demás, dando lugar a peleas, acusaciones, amenazas y no teniendo en cuenta sus sentimientos.

Las personas que utilizan un estilo pasivo/ inhibido no defienden sus intereses y derechos personales, no se preocupan de lo que piensan o sienten sino que, por el contrario, se muestran al servicio de la otra persona. Las personas 
pasivas/inhibidas priorizan las opiniones y los deseos de los demás por encima de los suyos propios. Esta forma de comportarse contribuye a que se generen sentimientos de resentimiento, irritación, culpa y/o ansiedad en la persona que adopta este estilo comunicativo.

El estilo asertivo es el estilo de comunicación más eficaz en la interacción. Las personas con tal estilo defienden sus propios intereses y derechos, expresando sus opiniones libremente y no dejando que los demás se aprovechen. A su vez, las personas que utilizan este estilo son capaces de respetar la forma de pensar del interlocutor, mostrándose considerados y respetuosos. Se adaptan a la situación, siendo capaces de aprender de ella y minimizar el riesgo de que puedan surgir problemas en interacciones futuras. El objetivo de comportarse de esta manera no es conseguir lo que se desea mediante la manipulación, sino comunicarse de forma clara y directa en base a lo que consideran sus objetivos. Las consecuencias de dicha forma de actuar son positivas, ya que permite controlar la situación, disminuir el estrés y respetarse a uno mismo, además de ser capaces de resolver problemas y sentirse bien con ellos mismos y con los demás.

Para mejorar la forma que tienen algunas personas de aprender a relacionarse de forma eficaz se utiliza el entrenamiento en habilidades sociales (EHS), que se basa en la teoría del aprendizaje social de Bandura (1977). El EHS es un tratamiento cognitivo conductual en el que se aplica un conjunto de técnicas cuyo objetivo es mejorar la calidad de las relaciones interpersonales, de comunicación y relacionales (Segrin, 2009).
Este entrenamiento se basa en técnicas de modificación de conducta, de la psicología social y de la teoría del aprendizaje social. Según Caballo (2002), el entrenamiento en habilidades sociales es una de las técnicas más eficaces y más frecuentemente utilizada en el tratamiento de problemas psicológicos, la mejora de la efectividad personal y la mejora de la calidad de vida.

En cuanto al contexto clínico, Méndez, Olivares y Ros (2010) destacan que el EHS se utiliza para problemas de comunicación con los hijos, tartamudez, fobias sociales, depresión, esquizofrenia, dificultades eróticas, problemas de pareja, abuso de tabaco, alcoholismo, obesidad y anorexia nerviosa.

Se considera entrenamiento asertivo a cualquier procedimiento terapéutico cuyo objetivo sea lograr que la persona, carente de habilidades asertivas, pueda reducir su ansiedad y conseguir manifestar conductas asertivas de forma socialmente apropiada. El entrenamiento asertivo es considerado hoy en día una de las estrategias de tratamiento más ampliamente utilizada dentro del marco de la salud mental (Caballo \& Irutia, 2004; Foster \& Bussman, 2008; Liberman, 2007; Mueser \& BeIlack, 2007; citados en Caballo \& Irurtia, 2008). Dicho entrenamiento consiste en eliminar del repertorio conductual de la persona aquellas conductas que se consideran no asertivas y dotarle de conductas asertivas para lograr una comunicación eficaz. Además, el entrenamiento asertivo permite reducir el estrés, enseñando a defender los legítimos derechos de cada uno sin agredir ni ser agredido (Davis, McKay, \& Eshelman, 1985). 
Con respecto a la eficacia del entrenamiento en asertividad, es de destacar que se considera uno de los tratamientos empíricamente validados según las guías de tratamientos eficaces (Gorman, 2002; Pérez, Fernández, Fernández, \& Amigo, 2003). Otros autores como Carrobles, Costa, Del Ser y Bartolome (1986) afirman que el entrenamiento asertivo es el método más eficaz y más utilizado para eliminar la ansiedad que muchas personas han aprendido a generar en situaciones interpersonales y que les impide comportarse adecuadamente en las mismas.

A continuación, se presenta un caso en el que se abordan las dificultades en una relación maternofilial, en el cual la consultante muestra un déficit de asertividad en las interacciones con su hija.

\section{Identificación de la paciente}

\section{y motivo de consulta}

M. es una mujer de 48 años, divorciada, sin pareja en la actualidad y que vive con su hija L., de 16 años. Indica que presentó una denuncia de malos tratos a su ex pareja, diagnosticado de esquizofrenia paranoide.

M. es funcionaria y trabaja como administrativa. Acude al Centro de Psicología Aplicada (en adelante CPA) y firma las normas de funcionamiento, entre las que se incluye el consentimiento del uso de sus datos para estudios científicos o estadísticos.

El objetivo que M. querría lograr es aprender a manejar los conflictos que tiene con su hija L. y así disminuir la tensión y el sufrimiento que ello le provoca; asimismo, refiere querer cambiar la sobreprotección que mantiene so- bre ella. Su principal problema es la mala relación que mantiene con su hija y las preocupaciones que tiene constantemente por ella, principalmente, por su futuro académico y por sus amistades. Ha comenzado a tener pensamientos disruptivos del tipo: "ojala se muera, cuánto la odio".

Lo que M. manifiesta querer conseguir durante el tratamiento es: "que mi hija entienda que no puede actuar así y que vea que no me desborda, no perder los papeles, no quiero estar encima". Se siente desanimada y sin ganas de hacer nada.

\section{Instrumentos y resultados de la evaluación}

Para la evaluación inicial del caso se utilizaron los siguientes instrumentos: entrevista personal, autorregistro básico de malestar, autorregistro de interacciones, historia clínica, cuestionario de ansiedad estado-rasgo (State-Trait Anxiety Inventory, STAI, de Spielberger, Gorsuch, \& Lushene, 1970; adaptación al español de Seisdedos , 2011) e, Inventario de Depresión de Beck (Beck Depression Inventory, BDI-II), de Beck, Steer y Brown, 1966; versión española, Sanz, Perdigón, \& Vázquez, 2003.

La entrevista personal se utilizó para recoger toda la información necesaria y hacer la formulación del caso mediante análisis funcional, que permitiera posteriormente diseñar un plan de tratamiento ajustado e individualizado. Se necesitaron dos sesiones, para evaluar los aspectos más importantes de la relación madre e hija. A la primera sesión acudieron ambas y se recabó información de cómo era su relación, la manera que tenían de comportarse y cómo ambas veían el problema. En la se- 
gunda sesión, se evaluó únicamente a M., centrando la atención en las interacciones con su hija, qué pensamientos tenía y qué hacía para afrontar su malestar (intentos de solución).

Se utilizaron autorregistros básicos para identificar las situaciones en las que M. sentía mayor malestar y autorregistros de interacciones (día, situación, ¿qué digo/ hago?, ¿qué dice/hace ella?) para obtener descripciones detalladas de los episodios en los que el problema se manifestaba. Por otra parte, se utilizaron estos autorregistros con el fin de identificar los estilos de comunicación (agresivo, asertivo, inhibido) adoptados por la paciente en las interacciones con su hija.

Los principales temas que se identificaron como desencadenantes de las discusiones fueron los amigos de L., temas políticos, hábitos de estudio e incumplimiento de obligaciones por parte de su hija. La frecuencia era diaria, especialmente por las mañanas, momento en el cual L. tenía que levantarse para ir a clase y M. se sentía responsable de su despertar. Los fines de semana aumentaba la frecuencia debido al malestar que M. sentía ante el incumplimiento de las normas que había tratado de imponer a su hija. Asimismo, la paciente describió sensaciones de angustia, rabia e impotencia en los momentos previos a cada desencuentro; opresión en el pecho y falta de aire durante los enfrentamientos y tener continuas ganas de llorar, tensión muscular y acusados dolores de cabeza tras los enfrentamientos con su hija.

Por otra parte, M. afirmó sentirse culpable, presentando atribuciones negativas o de autoculpabilidad con respecto a la conducta dis- ruptiva de su hija. Se observaron pensamientos recurrentes y persistentes de preocupación sobre el futuro de su hija, anticipando un posible abandono de sus estudios; asimismo, en su discurso se apreciaron ideas irracionales del tipo "debería". Además, se observó la creencia de M. de que su sufrimiento cesaría si su hija aceptara lo que ella le demandaba, por lo que sus intentos de solución consistían en intentar imponer su punto de vista de forma no asertiva, presionarla, insistir en lo que tiene que hacer, sobreprotegerla y no permitirle autonomía.

En el momento de la evaluación sus problemas familiares no parecían interferir en su área laboral; M. tiene un trabajo estable, trabaja como funcionaria en una administración del estado. Sin embargo, la esfera social sí se vio afectada, la consultante había reducido progresivamente la frecuencia de actividades gratificantes durante el fin de semana.

En lo que a datos familiares relevantes se refiere, M. describió a su madre como una buena persona, cariñosa y nerviosa. Asimismo, detalló la relación con su madre durante su infancia: "me repetía todo muchas veces llegando a ser muy pesada". En cuanto a su entorno social, el único lugar en el que se relacionaba con otras personas en el momento en que acude a terapia era su trabajo. En ese momento, M. no llevaba a cabo actividades de ocio, de manera que la mayor parte de su tiempo la dedicaba al trabajo o a realizar tareas domésticas.

Para evaluar el nivel de ansiedad, se utilizó el STAI, en su adaptación española, cuyos resultados obtenidos fueron un percentil 77 en la ansiedad estado y un percentil 75 en la ansiedad rasgo. 
Con el fin de evaluar el estado de ánimo, se administró el inventario BDI-II, cuya puntuación obtenida fue 3 , lo que corresponde a una depresión mínima.

Como resultado de la evaluación se concluye que la consultante presenta dificultades en el manejo de la relación con su hija. El problema parece relacionado con la sobreprotección de M. y su falta de asertividad en la interacción con la menor. Las situaciones conflictivas de gran intensidad y alta frecuencia llevaban a la paciente a perder el control, manifestando una sintomatología ansiosa.

\section{Análisis Funcional}

A raíz de la evaluación llevada a cabo se concluye que la consultante reacciona con hostilidad y malestar (respuesta condicionada o RC) ante las situaciones en las que su hija (estímulo condicionado o EC) está involucrada. No se identifica un origen claro más allá del cambio comportamental de la menor que se puede ver explicado por el comienzo de su adolescencia. Parece que se ha producido una generalización del malestar de la paciente a casi todos los aspectos de la convivencia con su hija. Por otro lado, el comportamiento de M. puede explicarse por el aprendizaje vicario de verbalizaciones insistentes por parte de su madre durante su infancia y de violencia verbal por parte de su ex marido en los años que duró su matrimonio. En algún momento actuar de esta forma con su hija pudo resultar funcional, siendo repetido en sucesivos ensayos y logrando el resultado deseado.

En cuanto al mantenimiento de la conducta problema, por un lado, se encuentran situa- ciones que no controla con su hija. Estas situaciones son el estímulo que antecede a la conducta de la paciente (estímulo discriminativo o ED) concretamente, que la paciente pierda el control, verbalizando en alto lo que piensa, yendo detrás de ella e insistiéndole, adoptando en la mayoría de las interacciones un estilo agresivo (respuesta operante o RO). En otras ocasiones, al tratar de interactuar con L. recibe agresiones verbales (castigo positivo o $\mathrm{C}+$ ) e incluso en alguna ocasión ha percibido que L. se ha autolesionado culpabilizándola $(C+)$, de manera que ha empezado incluso a manifestar conductas de evitación (RO). Como ejemplos de conductas de evitación se identifican: no poner límites, no cumplir con su palabra y ceder ante la conducta disruptiva de su hija, en lugar de comportarse de manera asertiva.

Una de las situaciones que más tensión le provoca es tener que despertar a su hija por las mañanas para que acuda puntual al instituto (ED), lo que genera en la paciente rumiaciones (RO) del tipo "tengo que estar pendiente de ella, vivo por y para ella". Es más, M. suele despertar a su hija (RO), consiguiendo reducir a corto plazo su malestar. Asimismo, y actuando de esta forma, M. no consigue que su hija aprenda a comportarse de manera autónoma, agravando su malestar y manteniendo el problema de relación entre ambas.

Todos estos comportamientos a corto plazo reducen su nivel de malestar (refuerzo negativo o R-) pero, a largo plazo, se traducen en un incremento de las interacciones conflictivas. Este tipo de situaciones repercuten en otros aspectos de la vida de M., como poder sentirse bien consigo misma. La sensación de inefi- 
cacia e incompetencia en la educación de su hija ha podido provocar una disminución de su autoestima. Por otra parte, la paciente ha dejado de realizar actividades que le resultaban gratificantes, como salir con sus amigos. En la Figura 1 se puede consultar un esquema de las principales cadenas de mantenimiento de la conducta de la consultante.

\section{Tratamiento}

Teniendo en cuenta la formulación clínica del caso, y acorde al análisis funcional, se acordaron los objetivos de tratamiento. Como objetivos generales se identifican los siguientes: (1) mejorar la relación materno-filial y (2) aumentar el estado de ánimo. Se pueden consultar los objetivos específicos y técnicas de

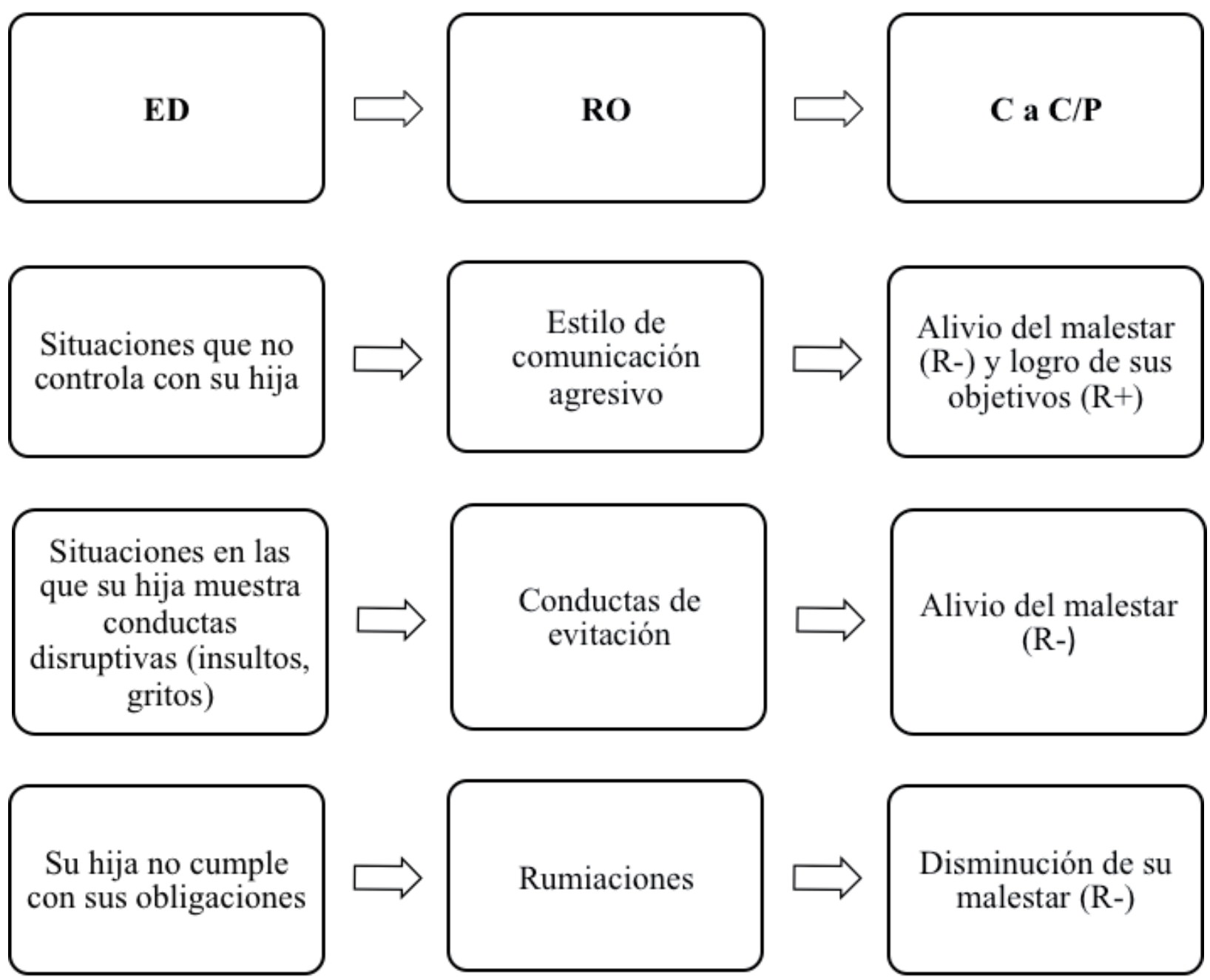

C a L/P: menor control en la educación de su hija, mantenimiento del malestar

Figura 1. Análisis funcional (principales hipótesis de mantenimiento). ED: estímulo discriminativo; RO: respuesta operante; R-: refuerzo negativo; $\mathrm{R}+$ : refuerzo positivo; $\mathrm{C}$ a $\mathrm{C} / \mathrm{P}$ : consecuencias a corto plazo; $\mathrm{C}$ a $\mathrm{L} / \mathrm{P}$ : consecuencias a largo plazo. 
Tabla 1

Técnicas de intervención y objetivos terapéuticos

Objetivos específicos

Reducir la frecuencia de intercambios aversivos entre ella y su hija.

Aumentar la frecuencia de intercambios agradables entre ella y su hija.

Adquirir habilidades de autocontrol.

Reducir su nivel de activación.

Aumentar la frecuencia de actividades gratificantes, sola o con amigos/familiares.
Técnicas

Entrenamiento en asertividad, entrenamiento en manejo del enfado y psicoeducación.

Planificación de actividades agradables.

Psicoeducación (curva del enfado) y entrenamiento en parada de pensamiento con autoinstrucciones.

Entrenamiento en respiración diafragmática y entrenamiento en autoinstrucciones.

Planificación de actividades agradables. intervención utilizadas para cada uno de ellos en la Tabla 1.

El tratamiento llevado a cabo incluyó técnicas cognitivo conductuales con el fin de que M. aprendiera a modificar su propio comportamiento, adaptándose a la situación aversiva y aprendiendo nuevas conductas y habilidades que le resultasen más eficaces.

El número de sesiones fue 25, de 50 minutos cada una, con una periodicidad semanal. El tratamiento se interrumpió durante los meses de verano, entre la sesión 12 y 13, por cierre del centro.

\section{Aplicación del tratamiento}

En la primera sesión acudieron a terapia madre e hija. El objetivo fue recoger información sobre su relación. En un primer momento entró a sesión L., quien dijo venir obligada por su madre, mostrándose muy reacia a recibir ayuda. M., por el contrario, se mostró muy colaboradora. Se identificaron los principales motivos de discusión, siendo estos los amigos de L., sus hábitos de estudio, la hora de levantarse para ir a clase y las malas contestaciones a su madre. El resto de la sesión se hizo de forma conjunta, explorando y observando la relación entre madre e hija y fomentando la reflexión sobre qué creían ambas que podrían cambiar.

En la segunda sesión, M. indicó que L. no iba a asistir a terapia. Se evaluaron las distintas áreas de la vida de la paciente, la topografía de sus conductas, su funcionalidad y los intentos previos de solución.

Durante el tiempo que duró la tercera sesión se explicó el análisis funcional con el objetivo de que $M$. entendiera porqué se estaba manteniendo su problema y se acordaron los objetivos terapéuticos. 
Con el objetivo de aumentar el estado de ánimo de M., en las sesiones cuarta y quinta se inició un programa de psicoeducación consistente en explicar a M. las características básicas psicológicas de la etapa de la adolescencia. Asimismo, se dedicaron estas sesiones al refuerzo del cumplimiento de actividades gratificantes y se planificaron otras nuevas, estableciéndose un compromiso de mantenimiento de las mismas. Se dio feedback sobre las últimas actuaciones con su hija, reforzando aspectos correctos de su desempeño e indicando que también había otros que se podían mejorar. En la quinta sesión, con el objetivo de adquirir habilidades de autocontrol, se ideó la curva del enfado para identificar los estímulos que discriminaban su ira y las estrategias que M. utilizaba para prevenir las discusiones y los desencuentros con su hija.

En la sexta sesión, se identificaron las dos situaciones que disparaban más habitualmente su enfado: que su hija le hablara constantemente de política y que se le hiciera tarde por las mañanas para llevar a su hija al instituto. Se identificaron y se reforzaron las estrategias que utilizaba para disminuir su activación: por un lado, decirle cómo se siente y, por otro, irse de la situación. Se facilitaron, además, otras recomendaciones para manejar las situaciones comentadas, como la extinción del comportamiento de su hija. Por otra parte, dado que M. anticipaba la situación temida de conflicto con su hija y esto le generaba cierta ansiedad que conllevaba una pérdida de autocontrol, se instruyó a M. a sustituir su diálogo interno negativo por autoinstrucciones positivas.
En la séptima y octava sesión se continuó dando feedback sobre su manera de actuar con L., comprometiéndose a no gritar y a no insistir, ya que su manera de actuar en un principio se caracterizaba por un estilo de comunicación agresivo; todo ello se hizo tanto en el comportamiento verbal (imposiciones, amenazas, ataques) como en el no verbal (voz alta, postura intimidatoria). Asimismo se dio feedback sobre la amenaza de imponer un castigo sin mantenerlo. En dichas sesiones, con el objetivo de aumentar su estado de ánimo se reforzó el cumplimiento de actividades agradables, así como el uso de autoinstrucciones positivas. En la octava sesión, se identificaron, además, las habilidades asertivas que M. querría aprender y resultase oportuno entrenar: hacer críticas, recibir críticas y negociar.

En la novena y décima sesión, se hizo una introducción sobre el concepto de asertividad para empezar con el entrenamiento y se entrenó a la paciente en la técnica de respiración diafragmática. Se retomó la curva del enfado con el objetivo de que identificase sus síntomas fisiológicos y cognitivos en momentos anteriores y posteriores al conflicto y se explicó en qué consiste la técnica de respiración controlada, cual es el objetivo y qué beneficios podía tener.

Durante la decimoprimera y decimosegunda sesión se reforzaron el uso de estrategias de afrontamiento adaptativas que M. estaba utilizando así como la importancia de favorecer la autonomía de su hija, reforzando los avances y las mejoras que M. había logrado. Se insistió en la importancia de que su hija se responsabilizara de las consecuencias de sus compor- 
tamientos y se abordó la conducta de sobreprotección de M., indicándole el efecto que su manera de actuar tenía en el comportamiento de su hija. Además, en estas sesiones, se evaluó si cumplía con la práctica de respiración y si lo hacía de la manera correcta, confirmando que había logrado en todos los ensayos reducir su nivel inicial de activación.

En la decimotercera sesión M. acudió a sesión tras las vacaciones de verano. Resumió cómo había ido la relación con su hija durante ese tiempo y se evaluó qué dificultades había encontrado y cómo había afrontado los problemas surgidos.

Se instruyó a M. en el uso de la técnica del disco rayado para ponerlo en práctica ante los desafíos de su hija, enseñándole a repetir su punto de vista de manera tranquila sin entrar en conflicto con la menor. El resto de sesión se dedicó a facilitar pautas educativas sobre cómo podría permitir a su hija tener independencia en sus decisiones. Se reforzó que M. siguiera manteniendo las conductas aprendidas en sesiones anteriores (extinguir las llamadas de atención de su hija, mantener el castigo que le impone y utilizar autoinstrucciones para fomentar sus habilidades de autocontrol).

La decimocuarta sesión se dedicó principalmente a permitir el desahogo emocional de M., ya que al inicio refirió haber tenido una semana "difícil" con su hija por su negativa a ir al instituto. Se reforzó haber utilizado la respiración diafragmática.

Dado los comportamientos retadores de su hija, se entrenó en solución de problemas y negociación. Para ello, se dio feedback sobre sus conductas y se hizo un modelado de cómo podría negociar con su hija las alternativas de solución.

Durante la decimoquinta sesión se reestructuraron sus ideas irracionales sobre la obligación que creía tener, de llamar todos los días a su hija por miedo a que dejara de ir al instituto. $M$. resumió qué es lo que le molestaba principalmente de este comportamiento de su hija y se identificaron las exigencias dogmáticas con respecto a cómo ella creía que debía comportarse su hija. Se identificaron las consecuencias negativas de estas exigencias y cómo repercutían en su conducta emocional y motora (enfados y discusiones).

La decimosexta y decimoséptima sesión se dedicaron a profundizar en el entrenamiento en asertividad con el objetivo de disminuir la frecuencia de intercambios aversivos. Se explicaron los tres estilos de respuesta (pasivo, agresivo y asertivo) y los derechos y responsabilidades asertivas. Asimismo, se reforzó que cada vez fuese más capaz de otorgar mayor independencia a su hija sin el sufrimiento que antes le generaba. A lo largo de estas últimas sesiones se confirmó el desempeño de actividades gratificantes con el objetivo de mejorar su estado de ánimo.

Finalmente, fue en la decimoctava y decimonovena sesión cuando se comenzaron a entrenar específicamente las habilidades asertivas evaluadas. Se entrenó a la paciente en la habilidad asertiva de hacer críticas. Se explicaron las instrucciones, se hizo un modelado negativo y otro positivo, así como un posterior ensayo de conducta. En primer lugar se llevó a cabo un ejemplo de situación neutra y posteriormente se utilizó un ejemplo de una situa- 
ción habitual en su convivencia con L., concretamente que no fuera capaz de levantarse por las mañanas de forma autónoma. Tras la ejecución de sus conductas se dio feedback y se reforzaron aquellas que fueron adecuadas.

Asimismo, se entrenó a la paciente en la habilidad asertiva de recibir críticas. Tras explicar las instrucciones se hicieron varios ensayos de conducta, tras los cuales se dio feedback y se reforzó su actuación. En todos los ensayos de conducta, llevados a cabo mediante roleplaying, se estableció la misma secuencia: modelado, instrucciones, reforzamiento y retroalimentación.

La última sesión de tratamiento se dedicó a evaluar los avances y el alcance de los objetivos terapéuticos, reconociendo ella misma su cambio conductual. Finalmente, se acordó comenzar el seguimiento, espaciando las sesiones en el tiempo.

\section{Seguimiento}

Se llevaron a cabo cinco sesiones de seguimiento con una periodicidad quincenal. Dichas sesiones se dedicaron principalmente a que la consultante explicitara cómo se comportaba con su hija. Se continuó dando feedback de sus actuaciones y se reforzó positivamente a M. por la asertividad que demostraba ante el comportamiento de la menor y en los límites que había sido capaz de imponer o negociar, así como concederle autonomía. Otro de los objetivos de estas sesiones fue confirmar el cumplimiento de actividades agradables.

\section{Resultados}

La evaluación de los resultados se realizó teniendo en cuenta datos de tipo cualitativo y cuantitativo. Los datos cualitativos sobre los resultados del tratamiento se obtuvieron a partir de verbalizaciones emitidas por la propia paciente, dedicando parte de la sesión 20 a evaluar los avances de M. y el alcance de los objetivos terapéuticos.

M. reconoce el cambio conductual que ha logrado y se confirma por verbalizaciones del tipo: "he cambiado en la manera de dirigirme a L., antes era más impulsiva y más agresiva, no gritar para mí es un logro", "antes también me salían insultos, ahora soy menos impulsiva, pienso un poco más", "he aprendido a respirar, autoinstrucciones, a no ser tan pesada con L., a dejarla a ella decidir", "en frecuencia han bajado los conflictos y en malestar también, ahora llegamos a acuerdos...".

Los datos cuantitativos se obtuvieron a partir del registro de la frecuencia de intercambios aversivos con su hija, evaluando y clasificando su forma de enfrentarse a la situación en los tres estilos de comunicación (agresivo, inhibido y asertivo). Asimismo, otros datos cuantitativos fueron obtenidos a partir de las puntuaciones de los cuestionarios administrados en el pretratamiento y postratamiento.

En cuanto al STAI, su Ansiedad Estado se redujo de un percentil 77 a un percentil 50 y su Ansiedad Rasgo de un percentil 75 a un percentil 20, lo que indica un cambio apreciable. Los resultados muestran que se ha producido una mejoría general, concretamente en las sensaciones de tensión manifestadas por la paciente, nerviosismo, preocupación y aprensión (véase Tabla 2). 
En cuanto a la administración del BDI-II, se debe puntualizar que proporciona una estimación global de la depresión. A pesar de la baja puntuación obtenida en la evaluación $(<10)$, se consideró relevante plantear la mejora del estado de ánimo como objetivo puesto que las verbalizaciones de la paciente al inicio de la intervención sí indicaban sintomatología depresiva. Además, se consideró relevante prestar atención a ítems específicos de tal cuestionario que pudieron ser determinantes a efectos clínicos. Estos ítems aportaron información sobre la consecución del objetivo general "aumentar estado de ánimo" y sobre el objetivo específico "aumentar la frecuencia de actividades agradables sola o con amigos/familiares".

En relación a la principal demanda de la paciente y su motivo de consulta (reducir la

Tabla 2

Puntuaciones obtenidas en el STAI

\begin{tabular}{lcccc}
\cline { 3 - 5 } & \multicolumn{2}{c}{ Pretratamiento } & \multicolumn{2}{c}{ Postratamiento } \\
\cline { 3 - 6 } & Punt. Directa & Percentil & Punt. directa & Percentil \\
\cline { 3 - 5 } STAI A/E & 27 & 77 & 15 & 50 \\
STAI A/R & 30 & 75 & 14 & 20 \\
\hline
\end{tabular}

\section{Interacciones madre-hija}

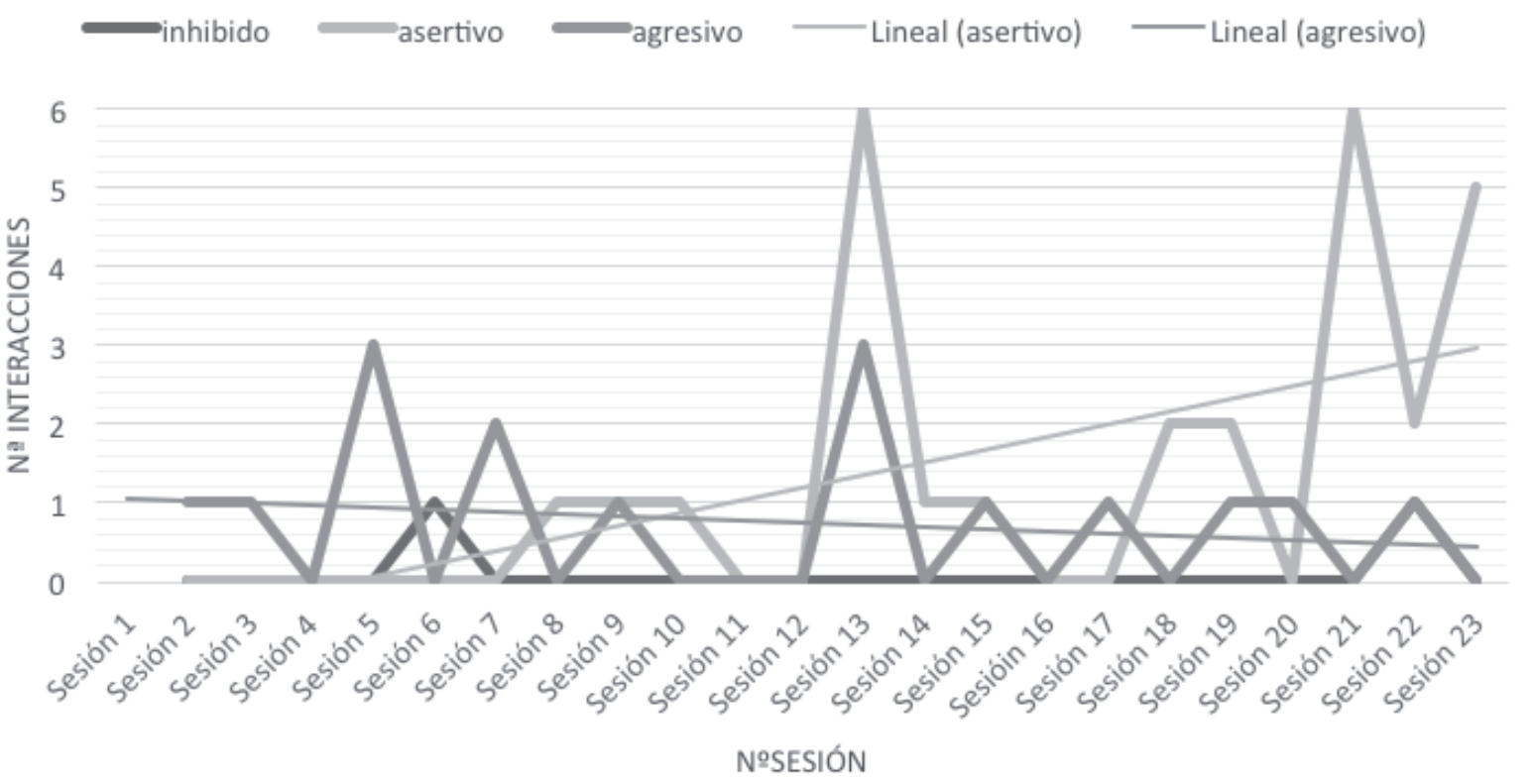

Figura 2. Registro de frecuencia de interacciones inhibidas, asertivas y agresivas semanales. Evaluación: sesiones 1,2. Explicación de análisis funcional: sesión 3. Tratamiento: sesiones de la 4 a la 20. Seguimiento: sesiones de las 21 a las 23 . Nota: No se registran las sesiones 24 y 25 porque se dedican a insistir en aspectos relevantes de modificación de conducta. 
frecuencia de intercambios aversivos), un indicador observable de mejoría fue el número de episodios semanales de comportamientos inhibidos, asertivos y agresivos evaluados mediante autorregistros semanales de interacciones (véase Figura 2).

El resultado fue un aumento de los episodios en los que la paciente consiguió poner en práctica la conducta asertiva aprendida, siendo 6 el número máximo de episodios asertivos. En la sesión 9 se explicó en qué consiste el comportamiento asertivo y parece que es a partir de esta sesión cuando empezó a mostrar cambios en cuanto a su forma de comportarse. Entre la sesión 12 y 13 de tratamiento se registró el máximo de episodios asertivos (6 episodios asertivos) y un total de 3 agresivos. Entre la sesión 20 y 21 (primera sesión de seguimiento) se registraron nuevamente 6 episodios asertivos con un total 0 episodios agresivos.

\section{Conclusiones}

En cuanto a los objetivos terapéuticos que M. alcanzó, es de destacar que son los acordados desde un principio, por lo que se considera una intervención eficaz. El único objetivo que no se ha conseguido es el referente a aumentar la frecuencia de intercambios agradables entre ella y su hija. Sin embargo, dado que su hija ha comenzado una terapia individual, se puede decir que es un primer paso para lograr tal avance.

Como queda reflejado en el apartado de resultados, se ha logrado una reducción de la frecuencia de los intercambios aversivos entre M. y su hija y se ha enseñado a manejar los conflictos de una forma adaptativa a través de técnicas de modificación de conducta (principalmente entrenamiento en asertividad, técnicas de control de la activación y estrategias de afrontamiento). Estas técnicas han ayudado a la paciente a disminuir su ansiedad y a controlar sus reacciones asociadas al estrés que la convivencia con su hija le provocaba, permitiéndole además aprender otra respuesta alternativa.

A nivel motor, es capaz de enfrentarse a dichas situaciones de manera adecuada, ya que ha aprendido a poner en práctica un estilo de comunicación asertivo.

A nivel fisiológico se ha reducido la sintomatología típica de episodios de ansiedad (tanto en Ansiedad Estado como en Ansiedad Rasgo) que al principio del tratamiento presentaba (tensión, nerviosismo, taquicardias).

Por último, en el área cognitiva, se ha observado un menor número de pensamientos negativos en la escalada de conflicto, que repercutían negativamente en su capacidad de afrontamiento en las situaciones ansiógenas.

Es de destacar que, en los resultados postratamiento del BDI-II se observa un ligero aumento de las puntuaciones correspondientes a los ítems 3 (sentimiento de fracaso) y 5 (sentimiento de culpa). Aunque puede resultar contradictorio que estas puntuaciones hayan aumentado tras el tratamiento, resulta relevante si se tiene en cuenta que tales sentimientos pueden deberse a la toma de conciencia del problema durante todo el tratamiento y a que cada vez más asume el papel activo y mantenedor que ella tiene en los conflictos con $\mathrm{L}$.

Siguiendo con la propuesta de objetivos, es importante comentar que antes de empezar el tratamiento se hizo especial hincapié en 
la posibilidad de incluir nuevos objetivos en cualquier momento de la terapia, dadas otras posibles variables influyentes o explicativas de su malestar (cáncer que sufrió, supuesto maltrato por su ex pareja, etc.). Aunque M. negó requerir otro tipo de ayuda, recibió la oferta de manera afable, verbalizando: "yo eso lo tengo superado, si me doy cuenta te lo diré". Aunque estos factores se consideraron al principio de la evaluación por si podían influir en el curso del tratamiento, la eficacia del tratamiento demuestra que no fueron tan relevantes.

En cuanto a las técnicas utilizadas, el entrenamiento en asertividad ha sido clave para lograr que el cambio de conducta de M. repercuta de manera positiva en las interacciones con su hija. Parece que el hecho de haber incidido en el aprendizaje de los estilos de comunicación y aprender a hacer/recibir críticas ha permitido que $M$. redujese la frecuencia de conductas de sumisión o enfrentamiento. La psicoeducación y la reestructuración de pensamientos irracionales también facilitaron el poder poner en práctica un estilo de comunicación asertivo.

En lo que a la mejora de su estado de ánimo se refiere, la programación de actividades es clave para mejorar los problemas psicológicos en general, disminuyendo los síntomas de ansiedad, de depresión o de cualquier otra manifestación psicológica que interfiera en su día a día (Gavino, 2008). En el caso de M., dado que su vida se había visto afectada por un déficit en el desempeño de actividades que ella misma considera gratificantes, fue necesario pautar actividades gratificantes como parte de la intervención psicológica, con el objetivo de retomar su vida cotidiana y disminuir los pensamientos disruptivos.

Como punto fuerte del trabajo se destacan las evaluaciones periódicas a lo largo de todo el proceso de intervención para comprobar si el tratamiento seguía la dirección adecuada. Durante todo el tratamiento, se dio gran importancia a los autorregistros (de interacciones y de autoinstrucciones). El uso de autorregistros y tareas para casa no sólo parecieron ser útiles para valorar el progreso, sino también para aumentar la percepción de M. de autoeficacia, al comprobar semanalmente sus logros.

Como posibles mejoras para futuros estudios similares se podrían considerar las siguientes: en primer lugar podría haberse utilizado el cuestionario de Perfil de estilos educativos (Magaz y García, 1998), concretamente el cuestionario de administración para padres, como una medida para obtener información más precisa sobre el resultado de la intervención y evaluar la eficacia del tratamiento. Este cuestionario hubiera permitido identificar y cuantificar las ideas, creencias, actitudes y valores correspondientes a un estilo educativo sobreprotector, inhibido, punitivo y asertivo.

Igualmente, pudo haber sido adecuada la administración de un cuestionario específico de asertividad, y así poder tener una medida pre-post adicional en este constructo. Sin embargo, no se consideró necesario puesto que la información recogida en la entrevista y los autorregistros fue suficiente para determinar las conductas en las que la paciente mostraba mayor dificultad. Como se ha observado en el apartado de resultados, fueron precisamente los autorregistros de interacciones los que per- 
mitieron cuantificar los estilos de comunicación utilizados y su evolución a lo largo del tiempo.

Por otra parte, se puede destacar que solo una de las partes del conflicto acude a consulta lo que, al tratarse de una relación interpersonal, hizo más difícil la consecución de los objetivos acordados. Durante el tratamiento se insistió en abordar los conflictos desde el entrenamiento en asertividad y un proceso de negociación y, considerando que una negociación requiere la participación de ambas partes del conflicto, el hecho de trabajar solo con $\mathrm{M}$. dificulta el proceso. La hija no quiso asistir a terapia en un primer momento, no reconocía su papel mantenedor y no se responsabilizaba de la situación ("mi madre está loca", "ella tiene la culpa", dijo). Desde el CPA se intentó contactar con L., para explicitar que podía volver al servicio cuando lo desease y lo considerase adecuado, pero resultó imposible lograr tal objetivo. Debido a la falta de asistencia de L., se advirtió de los límites de la terapia. No obstante, en el momento en que $M$. recibe el alta terapéutica, su hija L. decide comenzar una terapia en el centro.

Por último, y relacionado con lo anterior, es importante puntualizar que las relaciones interpersonales pueden tener efectos en la promoción y protección de la salud pero, en ocasiones, también pueden tener efectos perjudiciales para la salud.

Asimismo, un problema de relación padreshijos va asociado a un deterioro funcional en los dominios motores, cognitivos o emocionales. En el caso que nos ocupa, como ya se ha comentado, ejemplos de problemas motores son: inadecuado control, sobreprotección de la madre, presión materna excesiva, discusiones que se agravan hasta llegar a la amenaza de violencia física y la evitación sin la resolución de problemas. En lo que a problemas cognitivos se refiere es de destacar las atribuciones negativas a las intenciones de otro. Además, se aprecian problemas emocionales tales como sensación de tristeza, apatía y rabia contra el otro miembro de la relación. Por otra parte, en otras investigaciones, se ha señalado que el comportamiento perturbador de los menores puede generar sentimientos de ineficacia personal, estrés parental, frustración y rechazo en los padres, afectando negativamente a la relación padre-hijo y a la satisfacción familiar (Presentación-Herrero, Garcia, Miranda, Siegenthaler, \& Jara-Jiménez, 2006). Por todo ello, se considera fundamental seguir realizando estudios de este tipo que incrementen el conocimiento en este área de relaciones interpersonales entre distintas generaciones.

\section{Referencias}

Alberti, R. E., \& Emmons, M. L (1978). Your perfect right (3th ed). San Luis Obispo, California: Impact Press.

Bandura, A. (1977). Social learning theory. Englewood Cliffs, NJ: Prentice-Hall.

Beck, A. T., Steer, R. A., \& Brown, G. K. (1996). BDIII. Beck Depression Inventory- Second Edition. Manual. San Antonio, TX: The Psychological Corporation.

Bueno, M., \& Garrido M. (2012). Las relaciones interpersonales en la educación. Madrid: Pirámide.

Caballo, V. E. (1997). Manual de evaluación y entrenamiento en habilidades sociales (2. ${ }^{\mathrm{a}}$ ed.). Madrid: Siglo XXI.

Caballo, V. E. (2002). Manual de evaluación y entrenamiento en habilidades sociales (5. ${ }^{\mathrm{a}}$ ed.). Madrid: Siglo XXI.

Caballo, V. e Irurtia, M. J. (2008). Entrenamiento 
en habilidades sociales. En F. J., Labrador (Ed.), Técnicas de modificación de conducta (pp. 573-592). Madrid: Pirámide.

Carrascol. (1984). El entrenamiento en aserción. En J. Mayor, \& F. J. Labrador (Eds.), Manual de modificación de conducta (pp. 341-449). Madrid: Alhambra.

Carrobles, J. A., Costa, M., Del Ser, T., \& Bartolomé, P. (1986). La práctica de la terapia de conducta: teoría y método de aplicación para la práctica clínica. Valencia: Promolibro. Castanyer, O. (2014). Aplicaciones de la asertividad (2. ${ }^{\mathrm{a}}$ ed.). Bilbao: Desclée de Brouwer.

Davis, M., Eshelman, E. R., \& McKay, M. (1985). Técnicas de autocontrol emocional. Barcelona: Martínez Roca.

Gavino, A. (2008). Establecimiento de objetivos, planificación de actividades y administración del tiempo. En F. J. Labrador (Ed.), Técnicas de modificación de conducta (pp. 483-497). Madrid: Pirámide.

Gorman, N. (2002). A guide to treatments that work ( $2^{\mathrm{a}}$ ed). Nueva York, Estados Unidos: University Press.

Lazarus, A. (1966). Behavior rehearsal vs. Nondirective therapy vs. Advice in affecting behavior change. Behaviour Research and Therapy, 4, 209-212.

Llanos, C. (2006). Efectos de un programa de entrenamiento en habilidades sociales. (Tesis Doctoral). Departamento de Psicología Evolutiva y de la Educación, Universidad de Granada.

Magaz, A., \& García, E. M. (1998). Perfil de estilos educativos. Bizkaia: Albor-Cohs.

Méndez, F. X., Olivares, J., \& Ros, C. (2010). Entrenamiento en habilidades sociales. En J. Olivares, \& F.X., Méndez (Eds.), Técnicas de modificación de conducta (pp. 337-366). Madrid: Biblioteca Nueva.

Ortega, M., \& Calero, A. (2015). Un caso de interacción no asertiva. Revista Clínica Contemporánea, 6, 21-32.

Pérez, M., Fernández, J. R., Fernández, C., \& Amigo, I. (2003). Guía de tratamientos psicológicos eficaces I. Adultos. Madrid: Pirámide.

Presentación-Herrero, M. J., García Castellar, R.,
Miranda Casas, A., Siegenthaler Hierro, R., \& Jara-Jiménez, P. (2006). Impacto familiar de los niños con trastorno por déficit de atención con hiperactividad subtipo combinado: efecto de los problemas de conducta asociados. Revista de Neurología, $42,137-143$

Roca, E. (2007). Cómo mejorar tus habilidades sociales: Programa de asertividad, autoestima e inteligencia emocional (3. ${ }^{\mathrm{a}}$ ed.). Valencia: ACDE Psicología.

Salter, A. (1949). Conditioned reflex therapy: The direct approach to the reconstruction of personality. Nueva York: Creative Age Press.

Sanz, J., Perdigón, L. A., \& Vázquez, C. (2003). Adaptación sspañola del Inventario para la Depresión de Beck (BDI-II): 2. Propiedades psicométricas en población general. Clínica y Salud, 14, 249-280.

Segrin, G. (2009). Social skill Training. En W.T. O'Donohue, \& J.E. Fisjer (Eds.), General principles and empirically supported techniques of cognitive behaviour therapy. Hoboken, NJ: John Wiley and Sons.

Spielberger C., Gorsuch, R., \& Lushene, R. (1970). Manual for the State-Trait Anxiety Inventory. Palo Alto, California, Consulting Psychologist Press. (Adaptación Española, TEA, 2011). Versión española: Seisdedos, N. (2011), Madrid. TEA Ediciones.

Wolpe, J. (1958). Psychotherapy by Reciprocal Inhibition. Stanford, Estados Unidos: Stanford University Press. 
